\title{
Impact of Two Doses of Agonist Trigger on Assisted Reproductive Technology Outcome
}

\author{
Radha Vembu ${ }^{1}$, Sanjeeva R Nellepalli ${ }^{2}$, Monna Pandurangi ${ }^{3}$, Siddhartha Nagireddy ${ }^{4}$
}

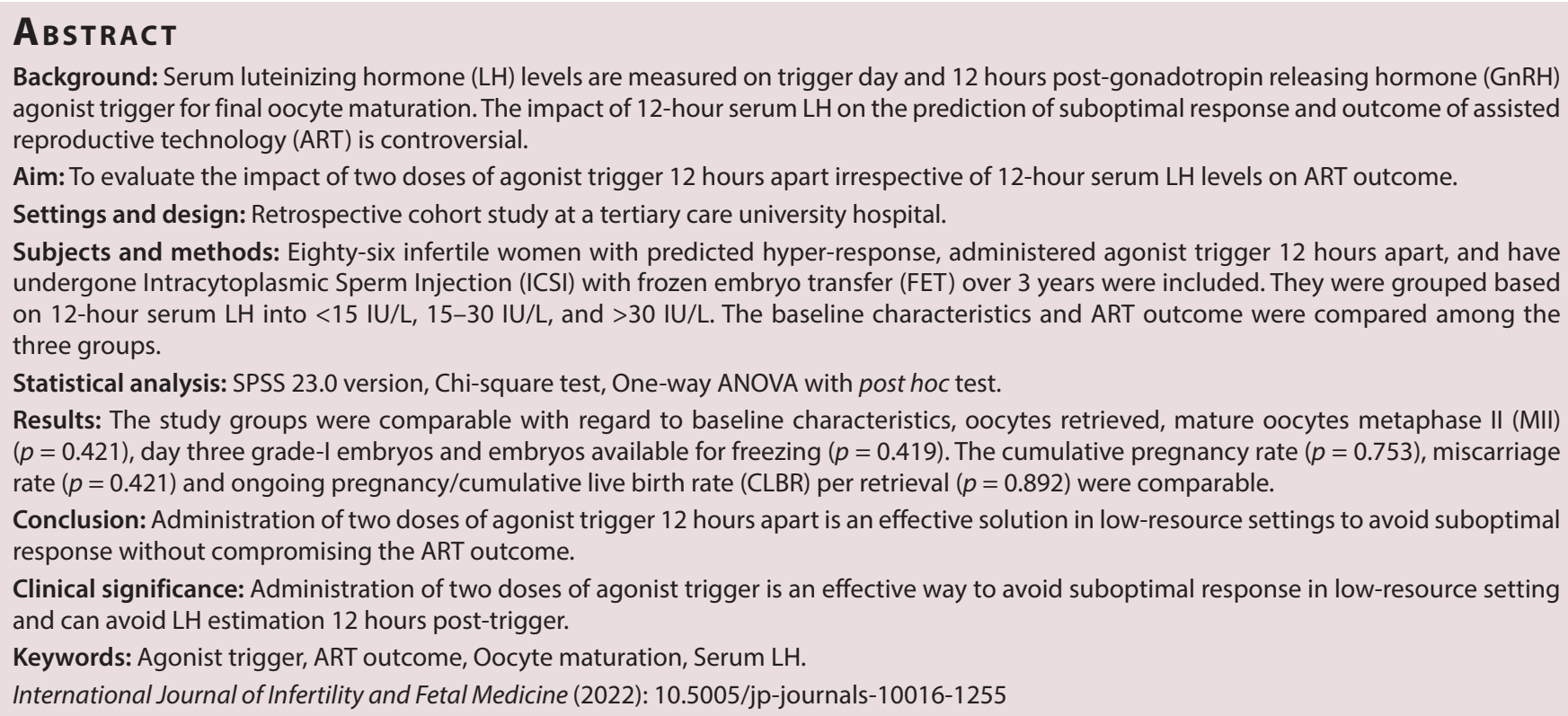

\section{INTRODUCTION}

Human chorionic gonadotropin (hCG) has been used as a surrogate for mid-cycle luteinizing hormone (LH) surge to induce final follicular maturation. The long half-life of hCG can lead to the dreaded complication of ovarian hyper stimulation syndrome (OHSS). It is proposed that administration of GnRH agonist trigger to induce final oocyte maturation will reduce the risk of OHSS. ${ }^{1}$ But the use of agonist trigger has been prevented by the fear and risk of some patients failing to respond adequately to this drug. ${ }^{2}$ There is a risk of suboptimal response (defined by 12 -hour LH post-trigger as $<15 \mathrm{IU} / \mathrm{L})^{3}$ due to reduced release of gonadotropins during the surge. This necessitates the need to asses LH level and administer the second dose of agonist or low dose hCG. However, there is time lag in the availability of laboratory parameters in a short duration to take further steps in most of the ART settings. There is not enough of data to suggest the ART outcome and Live Birth Rate (LBR) following agonist trigger. With this in background, a study was conducted to assess the number of mature oocytes retrieved and oocyte maturation rate (OMR) following administration of second dose of $\mathrm{GnRH}$ agonist irrespective of serum LH levels and compare the ART outcome in women with different levels of $\mathrm{LH}$, 12 hours post-trigger.

\section{Materials and Methods}

We conducted a retrospective cohort study of ICSI cycles from January 2016 to December 2019 in a tertiary care center. Infertile

\begin{abstract}
${ }^{1-4}$ Department of Reproductive Medicine \& Surgery, Sri Ramachandra Medical College and Research Institute, Sri Ramachandra University, Chennai, Tamil Nadu, India

Corresponding Author: Radha Vembu, Department of Reproductive Medicine \& Surgery, Sri Ramachandra Medical College and Research Institute, Sri Ramachandra University, Chennai,Tamil Nadu, India, Phone: +919841141310, e-mail: ganesh_radha@yahoo.in
\end{abstract}

How to cite this article: Vembu R, Nellepalli SR, Pandurangi M, et al. Impact of Two Doses of Agonist Trigger on Assisted Reproductive Technology Outcome. Int J Infertil Fetal Med 2022;13(1):1-4.

Source of support: Nil

Conflict of interest: None

women in the age-group of 21-42 years, identified as hyperresponders by ovarian reserve markers, anti-Mullerian Hormone $[A M H \geq 3.5 \mathrm{ng} / \mathrm{mL}$ and or antral follicle count (AFC) of $\geq 20,>14$ intermediary follicles and/or a high serum estradiol (E2) level $(>3500 \mathrm{pg} / \mathrm{mL}$ ] on the day of trigger, receiving two doses of agonist trigger 12-hour apart were included. Women with hypothalamic amenorrhea and hypogonadotropic-hypogonadism were excluded. So 86 women fulfilling the selection criteria were analyzed. Ovarian reserve assessment (determined by AMH levels, AFC on day 2 or 3 of menstrual cycle) was done after detailed history and clinical examination. 
On day 2 or 3 of menstrual cycle following suppression with combined pill (Ethinylestradiol $30 \mathrm{mcg}$ + Norethisterone $0.15 \mathrm{mg}$ ) for 15-21 days with pill free period of 5 days, all women underwent controlled ovarian stimulation (COS) with gonadotropins $\mathrm{rFSH}$ [Gonal-F, Merck] and or rFSH + HMG [Gynogen, Sanzyme] with flexible antagonist protocol. The dose, type of gonadotropin was decided based on age, ovarian reserve, $\mathrm{BMI}$, previous response to ovarian stimulation as per the institutional protocol. The starting dose varied from 150-225 IU/day. Following 5 days of gonadotropin injections, transvaginal ultrasound was performed and the dose of gonadotropins was adjusted based on the response. $\mathrm{GnRH}$ antagonist [Cetrorelix $0.25 \mathrm{mg}$ (Asporelix, Bharat serum \& vaccines)] was started when leading follicle was $\geq 14 \mathrm{~mm}$ or multiple follicles of $12 \mathrm{~mm}$ and continued along with gonadotropins till the day of ovulation trigger. When three or more follicles of $18-20 \mathrm{~mm}$, the decision for ovulation trigger was made. On the day of trigger, when multiple follicles of $>14 \mathrm{~mm}$ or serum estradiol level of $\geq 3500 \mathrm{pmol} / \mathrm{l}$, then $\mathrm{GnRH}$ agonist trigger in the form of injection Triptorelin $0.2 \mathrm{mg}$ [Decapeptyl, Ferring pharmaceuticals] subcutaneously, and 12 hours later, serum LH and progesterone (P4) were assayed by electro chemiluminescence immune assay. All study subjects received second dose of injection Triptorelin $0.1 \mathrm{mg}$ 12 hours after the first dose irrespective of the serum $\mathrm{LH}$ and P4 value. Oocyte retrieval was done 34-36 hours (first dose) after ovulation trigger under general anesthesia and ICSI was performed 2 hours postretrieval. Based on 12 hours post-trigger serum LH value, they were divided into three groups- $\mathrm{LH}<15 \mathrm{IU} / \mathrm{L}, 15-30 \mathrm{IU} / \mathrm{L}$, and $>30 \mathrm{IU} / \mathrm{L}$, and the data were analyzed in each group.

The following data were recorded namely the total dose of gonadotropins, duration of stimulation, number of follicles $>14 \mathrm{~mm}$ on the day of trigger, serum LH, and P4 on the day of trigger, and 12 hours post-trigger, number of oocytes retrieved, MIl oocytes, number of embryos (Grade I and II) on day 3, and grade I embryos as per Istanbul consensus. ${ }^{4}$

Follicle Oocyte Index (= ratio of total number of oocytes retrieved/AFC at the start of stimulation) and OMR (= percentage of the number of Mll oocytes to the total number of oocytes retrieved) were calculated as the all women had freeze all strategy to avoid the risk of OHSS, and FET was performed in suppressed cycle as per the institutional protocol.

On day 2 or 3 of next menstrual cycle, pituitary suppression was done with combined pill (Ethinyl estradiol $30 \mathrm{mcg}+$ Norethisterone $0.15 \mathrm{mg}$ ) and injection Leuprolide acetate depot $3.75 \mathrm{mg}$ subcutaneously. Following withdrawal bleeding on day 5 of cycle, hormone replacement therapy was started with estradiol valerate $4 \mathrm{mg}$ per day for 5 days followed by $6 \mathrm{mg}$ for 6 days. Transvaginal scan was performed to assess the endometrial thickness (ET) and pattern. The dose of estrogen was adjusted till the ET reached $8 \mathrm{~mm}$. Luteal support was started with injection micronized progesterone $100 \mathrm{mg}$ intramuscular/day, 3 days for cleavage embryos and 5 days for blastocysts till the day of embryo transfer and then changed to micronized vaginal progesterone $600 \mathrm{mg} /$ day along with tablet dydrogesterone $30 \mathrm{mg} /$ day till the day of serum $\beta$-hCG test. Luteal support was continued up to 10 weeks if pregnancy was confirmed. Transvaginal scan was performed for 2-3 weeks postembryo transfer to assess for the viability and at 11-14 weeks for nuchal translucency. All women were followed up for a maximum of two cycles of FET for conception and continued till delivery. Women who have conceived and delivered after previous transfer and wish to plan for another pregnancy and women who failed to conceive even after after two FETs were excluded from the study. The cumulative miscarriage rate (number of miscarriages/total number of pregnancy in two FET cycles), cumulative pregnancy rate (number of patients with $\beta$-hCG-positive/number of embryo transfers in two FET cycles, ongoing pregnancy rate (OPR) (pregnancy continuing beyond 20 weeks) and cumulative live birth rate (CLBR) is calculated as the number of deliveries that resulted in a live born neonate, expressed per 100 embryo transfers upto two FET cycles were included until the end of ART treatment or first live birth were recorded.

\section{Statistical Analysis}

The collected data were analyzed with IBM.SPSS statistics software 23.0 Version. To describe about the data descriptive statistics frequency analysis, percentage analysis was used for categorical variables and the mean and SD were used for continuous variables. To find the significant difference in the multivariate analysis, the one way ANOVA with Tukey's post hoc test was used. To find the significance in categorical data Chi-square test was used. In all the above statistical tools the probability value 0.05 are considered as significant level.

\section{Results}

All 86 women enrolled in the study were analyzed. Women in LH $<15 \mathrm{IU} / \mathrm{L}$ group were $16.3 \%, 15-30 \mathrm{IU} / \mathrm{L}$ were $26.7 \%$, and $>30 \mathrm{IU} / \mathrm{L}$ were $57 \%$. The mean age of the women was $28.8 \pm 3.8$ years, $68.6 \%$ of women had primary infertility and $66.3 \%$ had PCOS according to Rotterdam criteria. ${ }^{5}$ The mean basal LH was $6.4 \pm 3.6 \mathrm{IU} / \mathrm{L}$. The baseline characteristics were comparable in each group and are depicted in Table 1.

The mean total dose of gonadotropins, duration of stimulation, and number of follicles $>14 \mathrm{~mm}$ were compared in all the groups and were comparable without any significant difference (Table 2).

During COS, the hormonal levels of E2, LH, P4 on trigger day, LH, P4 12-hour post-trigger were measured among the three groups and were comparable (Table 3).

Comparison of LH and P4 on trigger day and 12-hour post-trigger among all study subjects showed a significant increase ( $p=0.0005)$ and the increase was 8.5 and 15.7-fold, respectively.

Table 1: Baseline characteristics

\begin{tabular}{|c|c|c|c|c|}
\hline Parameters & $<15$ IU/L (14) & $15-30 I U / L(23)$ & $>30 I U / L(49)$ & $p$-value \\
\hline Age (years) & $30.3 \pm 4.5$ & $28.2 \pm 2.8$ & $28.8 \pm 4.0$ & 0.253 \\
\hline Duration of infertility (years) & $7.0 \pm 4.5$ & $6.1 \pm 3.3$ & $5.5 \pm 3.6$ & 0.380 \\
\hline $\operatorname{BMI}\left(\mathrm{kg} / \mathrm{m}^{2}\right)$ & $25.8 \pm 4.3$ & $27.1 \pm 4.5$ & $25.6 \pm 4.3$ & 0.06 \\
\hline AFC & $28.9 \pm 14.1$ & $28.0 \pm 10.4$ & $24.0 \pm 8.6$ & 0.147 \\
\hline $\mathrm{AMH}(\mathrm{ng} / \mathrm{mL})$ & $7.5 \pm 4.0$ & $7.1 \pm 4.1$ & $7.0 \pm 3.6$ & 0.924 \\
\hline Baseline LH (IU/L) & $5.1 \pm 3.6$ & $6.3 \pm 4.4$ & $6.8 \pm 3.1$ & 0.276 \\
\hline
\end{tabular}


Among <15 IU/L group, there was 10.4 and 12.2-fold increase, respectively. Similarly, in 15-30 IU/L group, increase was 6.2 and 10.9-fold and in $>30 \mathrm{IU} / \mathrm{L}$ group, it was 8.8 and 24 -fold, respectively.

The outcome of COS like number of oocytes retrieved, MII, and the OMR showed no significant difference (Table 4).

The ART outcome following FET was analyzed and did not show any significant difference. In the subgroup analysis of patients with $\mathrm{LH}<0.5$ on trigger day $(N=23)$, the mean number of oocytes retrieved were $23.5 \pm 9.7$, Mll were $16.1 \pm 8.4$, and trigger day $\mathrm{P} 4$ were $1.5 \pm 0.9$ and 12-hour post-trigger P4 were $17.2 \pm 7.5$ which was similar to all the groups $(p=0.120)$.

\section{Discussion}

$\mathrm{GnRH}$ agonist has a shorter half-life which activates the $\mathrm{GnRH}$ receptors and induce release of Follicle stimulating hormone and LH similar to natural mid-cycle surge of gonadotropins. ${ }^{6}$ But this surge consists of only two phases namely ascending limb (4 hours) and a long descending limb (20 hours), in total of $24-36$ hours. $^{7}$ So the total amount of gonadotropin released during the surge is significantly reduced when compared to natural cycle. ${ }^{8,9}$ It is known that the expansion of cumulus cells and resumption of meiosis begin 18 hours of onset of LH surge ${ }^{10}$ and LH concentration should be maintained for 14-27 hours to maximize the oocyte maturation. ${ }^{11}$ So single dose of agonist trigger may not be enough to maintain the LH concentration. This short duration of action and the suboptimal response seen following single dose of agonist trigger in couple of patients in our center made us change to two doses of trigger followed in this study. To the best of our knowledge, this is one of the few studies of its kind comparing the COS and ART outcome following two doses of agonist trigger 12 hours apart irrespective of 12 hours LH levels post trigger. The assessment of LH in any laboratory requires around 3-6 hours especially in low resource setting. The decision to administer the second dose of either the agonist or hCG is delayed awaiting for the report.
This new protocol of two doses seems to be friendly to both clinician and patient by reducing the stress and anxiety of COS and avoid the risk of suboptimal response. This might influence all the clinicians to change their protocol.

Chang et al. found suboptimal response when 12 hour LH post trigger is $<15 \mathrm{IU} / \mathrm{L} .^{3}$ Whereas, Shapiro et al. found a value of $<52 \mathrm{IU} / \mathrm{L}^{12}$ and $40.69 \mathrm{IU} / \mathrm{L}$ in Deepika et al. study. ${ }^{13}$ We divided into THREE groups with $<15$ as cut-off as $16.3 \%$ of our study subjects were in this category.

In Chang et al. study, 38 out of 1,840 cycles failed to respond to agonist trigger and oocyte retrieval was done 36 hours after hCG trigger. $^{3}$ Even though it did not affect the clinical outcome in his study, it can affect the number of oocytes retrieved. In our study, we did not have any study subjects requiring a repeat procedure following two doses of agonist trigger.

The failure of agonist trigger is a nightmare to both clinician and the patient. It has been reported around $2-3.5 \%{ }^{14,15}$ The risk factors for this include low BMI, low baseline $\mathrm{LH}$, prolonged suppression with combined pills and use of higher dose of gonadotropins ( $>3800 \mathrm{IU}$ ). Women with $\mathrm{BMI}<22 \mathrm{~kg} / \mathrm{m}^{2}$ are twice at risk when compared to higher BMI. When we consider the baseline LH level, risk is $13.3 \%$ if $\mathrm{LH}$ is $<1 \mathrm{mlU} / \mathrm{mL}$ and $1.8 \%$ when $\mathrm{LH}$ is $\geq 2 \mathrm{mlU} / \mathrm{mL}$. The risk of cycle cancellation is $3.6 \%$ for $\mathrm{LH}$ value between 1 and $2 \mathrm{mIU} / \mathrm{mL}^{3}{ }^{3}$ In Popovic et al. study, serum basal LH of $<0.1 \mathrm{IU} / \mathrm{L}$ at the start of stimulation had a $45.2 \%$ risk of sub optimal response. ${ }^{16}$

In our study, we did not encounter this complication as the baseline characteristics and the hormonal profile were comparable in all the three groups with mean BMI of $26.64 .6 \mathrm{~kg} / \mathrm{m}^{2}$. These women received combined pills for pre cycle suppression for a maximum of three weeks and COS was started only after a wash out period of five days and the total dose of gonadotropin did not exceed $3000 \mathrm{IU}$.

The strength of this study is the assessment of serum LH exactly 12 hours post-trigger. This is important as early or late assay can increase or decrease the LH values, respectively. In Chang et al. study, ${ }^{3}$ LH was assayed between 8 and 13 hours post-trigger.

Table 2: Cycle characteristics

\begin{tabular}{lcccc}
\hline Parameters & $<15$ IU/L (14) & $15-30 /$ IU/L (23) & $>30 / U / L$ (49) & $p$-value \\
\hline Gonadotropin dose (IU) & $2827.7 \pm 1015.4$ & $2978.6 \pm 1150.7$ & $3056.6 \pm 1017.9$ & 0.253 \\
Duration of stimulation (days) & $10.3 \pm 1.8$ & $10.5 \pm 1.3$ & $10.0 \pm 1.3$ & 0.724 \\
Follicles $>14 \mathrm{~mm}$ & $20.4 \pm 6.0$ & $21.4 \pm 6.5$ & $19.7 \pm 7.1$ & 0.582 \\
\hline
\end{tabular}

Table 3: Hormonal characteristics during stimulation

\begin{tabular}{lcccc}
\hline Parameters & $<15$ IU/L (14) & $15-30 / \mathrm{IU} / \mathrm{L}(23)$ & $>30 / \mathrm{l} / \mathrm{L}(49)$ & $p$-value \\
\hline Trigger day E2 $(\mathrm{pg} / \mathrm{mL})$ & $5072.2 \pm 2768.0$ & $5019.9 \pm 2715.4$ & $5096.6 \pm 2845.7$ & 0.423 \\
Trigger day LH (IU/L) & $1.9 \pm 0.8$ & $2.1 \pm 1.6$ & $2.4 \pm 1.9$ & 0.724 \\
Trigger day P4 $(\mathrm{ng} / \mathrm{mL})$ & $1.4 \pm 0.8$ & $2.4 \pm 3.4$ & $1.6 \pm 1.1$ & 0.201 \\
12 hours-post-trigger P4 $(\mathrm{ng} / \mathrm{mL})$ & $14.2 \pm 9.5$ & $14.7 \pm 8.2$ & $14.0 \pm 6.6$ & 0.939 \\
\hline
\end{tabular}

Table 4: Outcome of controlled ovarian stimulation

\begin{tabular}{|c|c|c|c|c|}
\hline Parameters & $<15$ IU/L (14) & $15-30$ IU/L (23) & $>30 I U / L(49)$ & $p$-value \\
\hline Oocytes retrieved & $22.1 \pm 6.9$ & $23.0 \pm 10.9$ & $24.3 \pm 9.4$ & 0.703 \\
\hline M II & $13.6 \pm 4.2$ & $15.8 \pm 10.1$ & $16.9 \pm 7.9$ & 0.421 \\
\hline $\mathrm{FOl} *$ & $0.85 \pm 0.3$ & $0.88 \pm 0.4$ & $1.1 \pm 0.5$ & 0.07 \\
\hline OMR (\%) & $63.9 \pm 15.0$ & $69.2 \pm 23.5$ & $69.0 \pm 16.7$ & 0.626 \\
\hline Embryos for freezing & $10.1 \pm 3.2$ & $12.8 \pm 7.8$ & $14.2 \pm 6.9$ & 0.140 \\
\hline Day 3 Grade 1 & $6.4 \pm 3.5$ & $7.5 \pm 5.3$ & $8.6 \pm 6.0$ & 0.419 \\
\hline
\end{tabular}

* FOI (Follicle Oocyte Index = Ratio of total number of oocytes retrieved/AFC at the start of stimulation). 
This lead to unnecessary administration of hCG due to improper $\mathrm{LH}$ value and increased the risk of OHSS.

In addition to serum LH, serum P4 estimation helps in suspecting suboptimal response. The concentration of $\mathrm{P} 4$ reaches $3 \mathrm{ng} / \mathrm{mL}$ by 6 hours of agonist trigger. But it is also influenced by the number of follicles as patients with less number of follicles have less luteinization and hence low P4. ${ }^{7}$ Kummer et al., in his study, evaluated the value of serum $\mathrm{LH}$ and $\mathrm{P} 4$ post-trigger to predict the oocyte yield. Although there is no cut-off to predict the number of oocytes retrieved, all patients with empty follicle syndrome (EFS) had low serum LH levels of $15 \mathrm{IU} / \mathrm{L}$ and progesterone levels of $\leq 3.5 \mathrm{ng} / \mathrm{mL}$. $^{15}$ This is useful for counseling the patients about the chances of EFS. In our study, $16.4 \%$ had LH $<15 \mathrm{IU} / \mathrm{L}$ and we observed an 8.5 and 15.7 -fold increase in serum LH and P4 after 12 hours of agonist trigger among all study subjects. When we compared in all the three groups, the increase was statistically significant. So, P4 is another marker of adequate ovarian response.

In our study, even though the risk of failure of trigger is less, additional dose of agonist trigger 12 hours later simplified the protocol and outcome of $\operatorname{COS}$ which is applicable to all low resource settings. This reduces the stress and anxiety to both clinician and the patient and is more economical, reduces the risk of OHSS secondary to administration of hCG as trigger to rescue the cycle.

In the present study, we planned for freeze all strategy as per our protocol to avoid the poor outcome of poor luteal support and the risk of delayed OHSS. Yousef et al. in their systematic review and meta analysis found that when GnRH agonists is used for final oocyte maturation in fresh autologous cycles, it was associated with a significantly lower LBR, lower OPR, and higher rate of early miscarriage. ${ }^{17}$ In Deepika et al. study, they observed that two doses of agonist trigger 12 hours apart provided a better outcome than single dose in terms of mature oocytes, number of blastocysts, and clinical pregnancy rate. ${ }^{13}$ But in their study, they did not compare the outcome based on the LH values and OPR/LBR. This is another strength of our study where we compared the COS and ART outcome in all the groups including CLBR. In our study, the cumulative pregnancy rate, miscarriage rate, and OPR/CLBR were similar in all the groups. This implies that second dose of agonist did not affect the quality in all the three groups and had similar number of embryos available including grade I embryos in all the three groups (statistically not significant) and indirectly improved the cumulative ART outcome. The limitation of the study is retrospective study design and smaller sample size. We did not assess the LH level after second dose of agonist trigger that is prior to oocyte retrieval in view of the additional cost.

\section{Conclusion}

Administration of two doses of agonist trigger 12 hours apart is an effective solution in low resource settings to avoid suboptimal response without compromising the ART outcome.

\section{References}

1. Itskovitz-Eldor J, Kol S, Mannaerts B. Use of a single bolus of $\mathrm{GnRH}$ agonist triptorelin to trigger ovulation after $\mathrm{GnRH}$ antagonist ganirelix treatment in women undergoing ovarian stimulation for assisted reproduction, with special reference to the prevention of ovarian hyper- stimulation syndrome: preliminary report: short communication. Hum Reprod 2000;15:1965-1968. DOI: 10.1093/humrep/15.9.1965
2. Honnma $\mathrm{H}$, Hashiba $\mathrm{Y}$, Asada $\mathrm{Y}$, et al. Failure of triggering oocyte maturation with a GnRH agonist in polycystic ovary syndrome: two case reports. Eur J Obstet Gynecol Reprod Biol 2011;157:239-240. DOI: 10.1016/j.ejogrb.2011.03.002

3. Chang FE, Beall SA, Cox JM, et al. Assessing the adequacy of gonadotropin-releasing hormone agonist leuprolide to trigger oocyte maturation and management of inadequate response. Fertil Steril 2016;106(5):1093-1100.e3. DOI: 10.1016/j.fertnstert.2016.06.013

4. ALPHA Scientists in Reproductive Medicine, ESHRE Special Interest Group Embryology. Istanbul consensus workshop on embryo assessment: proceedings of an expert meeting. Reprod Biomed Online 2011;22:632-646. DOI: 10.1093/humrep/der037

5. Azziz R. Controversy in clinical endocrinology: diagnosis of polycystic ovarian syndrome: the Rotterdam criteria are premature. J Clin Endocrinol Metab 2006;91:781-785. DOI: 10.1210/jc.2005-2153

6. Nakano RT, Mizuno F, Kotsuji K et al. "Triggering" of ovulation after infusion of synthetic luteinizing hormone releasing factor (LRF). Acta Obstet Gynecol Scand 1973;52:269-272. DOI: $10.3109 / 00016347309158325$

7. Itskovitz J, Boldes R, Levron J, etal. Induction of preovulatory luteinizing hormone surge and prevention of ovarian hyperstimulation syndrome by gonadotropin-releasing hormone agonist. Fertil Steril 1991;56:213-220. PMID: 1906406.

8. Hoff JD, Quigley ME, Yen SS. Hormonal dynamics at mid cycle: a reevaluation. J Clin Endocrinol Metab 1983;57:792-796. DOI: 10.1210/jcem-57-4-792

9. Zelinski-Wooten MB, Lanzendorf SE, Wolf DP, et al. Titrating luteinizing hormone surge requirements for ovulatory changes in primate follicles. I. Oocyte maturation and corpus luteum function. J Clin Endocrinol Metab 1991;73:577-583. DOI: 10.1210/jcem-73-3-577

10. Seibel MM, Smith DM, Levesque L, et al. The temporal relationship between the luteinizing hormone surge and human oocyte maturation. Am J Obstet Gynecol 1982;142:568-572. DOI: 10.1016/0002-9378(82)90763-3

11. Zelinski-Wooten MB, Hutchison JS, Chandrasekher YA, et al. Administration of human luteinizing hormone $(h L H)$ to macaques after follicular development: further titration of LH surge requirements for ovulatory changes in primate follicles. J Clin Endocrinol Metab 1992;75(2):502-507.DOI: 10.1210/jcem.75.2.1639951

12. Shapiro BS, Daneshmand ST, Restrepo H, et al. Efficacy of induced luteinizing hormone surge after "trigger" with gonadotropin-releasing hormone agonist. Fertil Steril 2011;95:826-828. DOI: 10.1016/j. fertnstert.2010.09.009

13. Deepika K, Baiju P, Gautham P, et al. Repeat dose of gonadotropin-releasing hormone agonist trigger in polycystic ovarian syndrome undergoing in vitro fertilization cycles provides a better cycle outcome - a proof-of-concept study. J Hum Reprod Sci 2017;10:271-280. DOI: 10.4103/jhrs.JHRS_102_17

14. Griesinger G, Schultz L, Bauer T, et al. Ovarian hyperstimulation syndrome prevention by gonadotropin-releasing hormone agonist triggering of final oocyte maturation in a gonadotropin-releasing hormone antagonist protocol in combination with a "freeze-all" strategy: a prospective multicentric study. Fertil Steril 2011;95:2029-2033. DOI: 10.1016/j.fertnstert.2011.01.163

15. Kummer NE, Feinn RS, Griffin DW, et al. Predicting successful induction of oocyte maturation after gonadotropin-releasing hormone agonist (GnRHa) trigger. Hum Reprod 2013;28:152-159. DOI: 10.1093/humrep/des361

16. B. Popovic-Todorovic B, Santos-Ribeiro S, Drakopoulos P, et al. Predicting suboptimal oocyte yield following GnRH agonist trigger by measuring serum $\mathrm{LH}$ at the start of ovarian stimulation. Hum Rep 2019;34:2027-2035. DOI: 10.1093/humrep/dez132

17. Youssef MAF, Abdelmoty HI, Ahmed MAS, et al. GnRH agonist for final oocyte maturation in GnRH antagonist co-treated IVF/ICSI treatment cycles: systematic review and meta-analysis. J Adv Res 2015;6:341-349. DOI: 10.1016/j.jare.2015.01.005 